\title{
Nonlinear oscillations of laminated plates using an accurate four-node rectangular shear flexible material finite element
}

\author{
GAJBIR SINGH* and G VENKATESWARA RAO \\ Structural Engineering Group, Vikram Sarabhai Space Centre, \\ Trivandrum 695022 , India \\ *Present address: Structures Group, ISRO Satellite Centre, Bangalore 560017 , \\ India \\ e-mail: gajbir@isac.ernet.in; gv-rao@vssc.org
}

\begin{abstract}
The objective of the present paper is to investigate the large amplitude vibratory behaviour of unsymmetrically laminated plates. For this purpose, an efficient and accurate four-node shear flexible rectangular material finite element (MFE) with six degrees of freedom per node (three displacements $(u, v, w)$ along the $x, y$ and $z$ axes, two rotations $\left(\theta_{x}\right.$ and $\left.\theta_{y}\right)$ about $y$ and $x$ axes and twist $\left(\theta_{x y}\right)$ ) is developed. The element assumes bi-cubic polynomial distribution with sixteen generalized undetermined coefficients for the transverse displacement. The fields for section rotations $\theta_{x}$ and $\theta_{y}$, and in-plane displacements $u$ and $v$ are derived using moment-shear equilibrium and in-plane equilibrium equations of composite strips along the $x$ - and $y$-axes. The displacement field so derived not only depends on the element coordinates but is a function of extensional, bending-extensional coupling, bending and transverse shear stiffness as well. The element stiffness and mass matrices are computed numerically by employing $3 \times 3$ Gauss-Legendre product rules. The element is found to be free of shear locking and does not exhibit any spurious modes. In order to compute the nonlinear frequencies, linear mode shape corresponding to the fundamental frequency is assumed as the spatial distribution and nonlinear finite element equations are reduced to a single nonlinear second-order differential equation. This equation is solved by employing the direct numerical integration method. A series of numerical examples are solved to demonstrate the efficacy of the proposed element.
\end{abstract}

Keywords. Nonlinear oscillations; unsymmetrically laminated plates; finite element method; direct numerical integration method.

\section{Introduction}

Fibre-reinforced composites, due to their high specific strength and stiffness and their anisotropic properties, which can be tailored depending on the design requirement, are fast replacing the traditional metallic structures in the weight-sensitive aerospace and aircraft industries. These structures invariably experience severe dynamic environments during 
their service; thus the excited motions are likely to have large amplitudes. The large amplitude analysis of composite structures is far more complex due to (i) anisotropy, (ii) material couplings, and (iii) more pronounced transverse shear flexibility effects compared to their isotropic counterparts. These structures with complex boundary conditions, loadings and shapes are not easily amenable to analytical solutions and hence one has to resort to numerical methods such as finite elements (Zienkiewicz 1977; Bathe 1990). A considerable amount of effort has gone into the development of simple plate bending elements based on the Yang, Norris and Stavsky theory (Yang et al 1966) which is a consistent extension of Mindlin's theory for homogeneous isotropic plates. The advantages of this approach are (i) it accounts for transverse shear deformation, (ii) it requires only $C^{0}$ continuity of the field variables, and (iii) it is possible to develop finite elements based on six engineering degrees of freedom viz. three translations and three rotations (see Ahmed et al 1970). However, the low-order elements, i.e. the 3-node triangular, 4-node and 8-node quadrilateral elements, locked and exhibited violent stress oscillations. Many techniques have been tried to overcome this, with varying degrees of success. The most prevalent technique to avoid shear locking for such elements is a reduced or selective integration scheme (Pawsey \& Clough 1971; Zienkiewicz et al 1971; Hughes et al 1977; Pugh et al 1978). The other notable successes are hybrid and mixed methods (Noor \& Anderson 1977; Lee \& Pian 1978; Spilker \& Munir 1980), modified shear strain methods (Hughes \& Tezduyar 1981; Crisfield 1984) and field consistency (Prathap 1984, 1985; Somashekar et al 1987) approach. In all these studies (Pawsey \& Clough 1971; Zienkiewicz et al 1971; Hughes et al 1977; Noor \& Anderson 1977; Lee \& Pian 1978; Pugh et al 1978; Spilker \& Munir 1980; Hughes \& Tezduyar 1981; Crisfield 1984), shear stresses at nodes are inaccurate and need to be sampled at certain optimal points derived from considerations based on the employed integration order (Hinton \& Campbell 1974). The use of the same interpolation functions for transverse displacement and section rotations in these elements results in a mismatch of the order of polynomial for the transverse shear strain field. This mismatch in the order of polynomials is responsible for shear locking. Techniques like reduced/selective integration do alleviate this problem of shear locking, but, in certain cases, zero energy spurious modes get introduced.

Nonlinear or large amplitude vibrations of beams and plates have been investigated extensively during the last few decades (refer, the comprehensive survey by Sathyamoorthy 1982, 1987 and standard text books, Stoker 1950, Nayfeh \& Mook 1979). Nonlinear vibrations of beams and plates still continue to interest researchers as reliable predictions of large amplitude motions are of great importance in avoiding catastrophic failures (Huang 1998). As a consequence, new/different techniques are being attempted to study the phenomenon (Leung \& Chui 1997; Qaisi 1997).

Till date, the finite elements employed for the nonlinear vibration analysis of beams and plates are based on independent polynomials of same order for all the field variables. This type of elements with low-order of interpolation for field variables exhibit shear locking in the case of thin plates if the associated element matrices are integrated exactly. Though quite a few approaches have been proposed over the years to eliminate the locking associated problems caused by the independent and same-order polynomial description for all field variables, a possible alternative displacement field has received little attention. The authors, in their quest for an alternative displacement field, realised the fact that in a flexural motion the transverse displacement and section rotations are always coupled even for homogeneous isotropic plates. In the case of an unsymmetrically laminated plate, the in-plane and out-of-plane responses are also coupled. As mentioned earlier, the coupling in 
transverse displacement and bending rotation through transverse shear strain has resulted in shear locking in the lower order $C^{0}$ elements. The performance of these $C^{0}$ elements further deteriorates when in-plane and out-of-plane field variables are also coupled due to material asymmetry. This made us believe that a properly derived, coupled displacement field would render an efficient, accurate and lock-free plate bending element. This paper is a modest attempt towards this endeavour. The displacement field has been derived using equilibrium equations and is found to be a function of various stiffnesses apart from the usual element geometry. To distinguish this class of elements from the conventional ones, the authors felt it appropriate to classify them as Material Finite Elements (MFE). In order to compute the nonlinear frequencies, linear mode shape corresponding to the fundamental frequency is assumed as the spatial distribution and nonlinear finite element equations are reduced to a single nonlinear second-order differential equation. This equation is solved by employing the direct numerical integration method. A series of numerical examples are solved to demonstrate the efficacy of the proposed MFE element over a wide range of plate configurations.

\section{Governing equations}

A rectangular plate composed of perfectly bonded layers of length $a$, width $b$ and total thickness $t$ as shown in figure 1 is considered. Each layer is made up of unidirectional fibres and is assumed to be a homogeneous orthotropic lamina. The orthotropic axes of symmetry in each lamina of arbitrary thickness and elastic properties are oriented at an arbitrary angle $\alpha$ with the $x$-axis of the plate. The components of displacement at a generic point in the plate are expressed in the form:

$$
\begin{aligned}
U(x, y, z, \tau) & =u(x, y, \tau)+z \theta_{x}(x, y, \tau), \\
V(x, y, z, \tau) & =v(x, y, \tau)+z \theta_{y}(x, y, \tau), \\
W(x, y, z, \tau) & =w(x, y, \tau) .
\end{aligned}
$$

In (1), $u, v$ and $w$ are displacements of the corresponding point on the middle plane and $\theta_{x}$ and $\theta_{y}$ are rotations of the normal to middle plane about the $y$ and $x$ axes respectively.

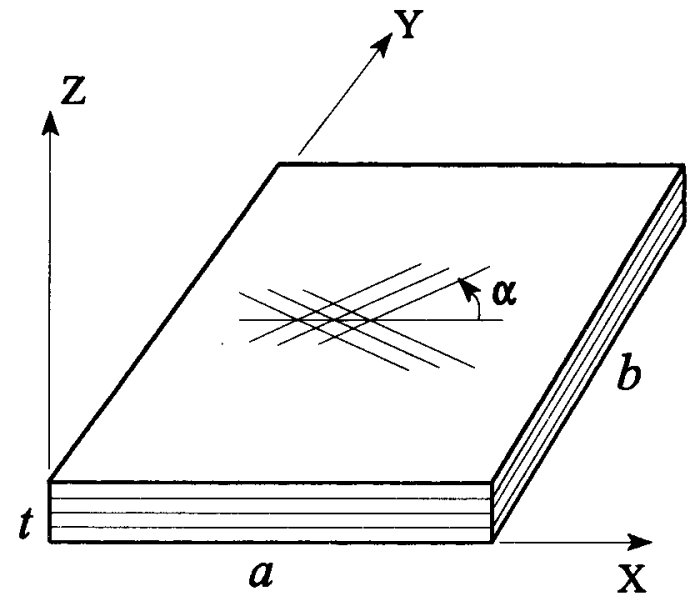

Figure 1. Geometry of a laminated plate. 
The nonlinear membrane strains $\left(\epsilon_{x x}, \epsilon_{y y}, \gamma_{x y}\right)$, curvatures $\left(\kappa_{x x}, \kappa_{y y}, \kappa_{x y}\right)$ and shear strains $\left(\gamma_{x z}, \gamma_{y z}\right)$ are related to the displacements and rotations by the following equations.

$$
\begin{aligned}
& \{\epsilon\}=\left\{\begin{array}{l}
\epsilon_{x x} \\
\epsilon_{y y} \\
\gamma_{x y}
\end{array}\right\}=\left\{\begin{array}{c}
u, x+\frac{1}{2} w,_{x}^{2} \\
v, y+\frac{1}{2} w,_{y}^{2} \\
u, y+v,,_{x}+w,,_{x} w, y
\end{array}\right\}, \\
& \{\kappa\}=\left\{\begin{array}{l}
\kappa_{x x} \\
\kappa_{y y} \\
\kappa_{x y}
\end{array}\right\}=\left\{\begin{array}{c}
\theta_{x, x} \\
\theta_{y, y} \\
\theta_{x, y}+\theta_{y, x}
\end{array}\right\} ; \\
& \{\gamma\}=\left\{\begin{array}{l}
\gamma_{x z} \\
\gamma_{y z}
\end{array}\right\}=\left\{\begin{array}{l}
w,_{x}+\theta_{x} \\
w,_{y}+\theta_{y}
\end{array}\right\} .
\end{aligned}
$$

The membrane stress resultants $\left(N_{x x}, N_{y y}, N_{x y}\right)$, stress couples $\left(M_{x x}, M_{y y}, M_{x y}\right)$ and transverse shear forces $\left(Q_{x z}, Q_{y z}\right)$ in a composite plate are related to the membrane strains, curvatures and transverse shear strains by the following constitutive relations.

$$
\begin{aligned}
& \{N\}=[A]\{\epsilon\}+[B]\{\kappa\}, \\
& \{M\}=[B]\{\epsilon\}+[D]\{\kappa\}, \\
& \{Q\}=[S]\{\gamma\} .
\end{aligned}
$$

In (5)-(7), $A_{i j}, B_{i j}$ and $D_{i j}(i, j=1,2,6)$ are the usual extensional, bending-extensional coupling and bending -stiffness coefficients of the composite laminate; $S_{i j}(i, j=4,5)$ are the transverse shear stiffness coefficients of the laminate and include $5 / 6$ as the shear correction factors.

The potential energy functional, neglecting in-plane inertia, is given by

$$
\begin{gathered}
\prod\left(u, v, w, \theta_{x}, \theta_{y}\right)=\frac{1}{2} \int_{\Omega}\left[\{\epsilon\}^{T}[A]\{\epsilon\}+\{\epsilon\}^{T}[B]\{\kappa\}+\{\kappa\}^{T}[B]\{\epsilon\}+\{\kappa\}^{T}[D]\{\kappa\}\right. \\
\left.+\{\gamma\}^{T}[S]\{\gamma\}+R_{r} \dot{\theta}_{x}^{2}+R_{r} \dot{\theta}_{y}^{2}+R_{t} \dot{w}^{2}\right] \mathrm{d} \Omega
\end{gathered}
$$

where $R_{r}$ and $R_{t}$ are the rotational and translational inertia and $\Omega$ is the domain of the plate. The in-plane inertia is neglected based on the study (Singh \& Rao 1998) which indicates that the influence of axial inertia on the linear and nonlinear flexural frequencies of symmetrically and unsymmetrically laminated beams is negligibly small.

\section{Material finite element formulation (MFE)}

The plate region $\Omega$ is decomposed into four-node rectangular finite elements having subdomain $\Omega_{\boldsymbol{e}}$ and interconnected at the four corners. Let the Cartesian coordinates of the nodes be $\left(x_{1}, y_{1}\right),\left(x_{2}, y_{2}\right),\left(x_{3}, y_{3}\right)$ and $\left(x_{4}, y_{4}\right)$ respectively. Among the five fundamental unknowns $u, v, w, \theta_{x}$ and $\theta_{y}$, the transverse displacement field $w$ is approximated by the complete bi-cubic as:

$$
\begin{aligned}
w= & c_{1}+c_{2} x+c_{3} y+c_{4} x^{2}+c_{5} x y+c_{6} y^{2}+c_{7} x^{3}+c_{8} x^{2} y+c_{9} x y^{2}+c_{10} y^{3} \\
& +c_{11} x^{3} y+c_{12} x^{2} y^{2}+c_{13} x y^{3}+c_{14} x^{3} y^{2}+c_{15} x^{2} y^{3}+c_{16} x^{3} y^{3}
\end{aligned}
$$


This field description was employed by Bonger et al (1966) and Singh et al (1993) to develop high precision $C^{\mathrm{l}}$ continuous elements for homogeneous isotropic thin and laminated thick plates respectively. These studies indicate that complete bi-cubic approximation for transverse displacement, $w$, leads to an accurate and efficient element. However, the accuracy is at the cost of a larger number of degrees of freedom per node.

\subsection{Field for in-plane displacement $u$ and rotation $\theta_{x}$}

To derive the field for in-plane displacement $u$ and section rotation $\theta_{x}$, the equilibrium of a strip along $x$-axis is considered. The equilibrium equations of the strip are obtained from plate equilibrium equations by dropping terms involving derivatives with respect to $y$. These simplified equations are:

$$
\begin{aligned}
& A_{11} u, x x+A_{16} v,_{x x}+B_{11} \theta_{x, x x}+B_{16} \theta_{y, x x}=0, \\
& A_{16} u, x x+A_{66} v,_{x x}+B_{16} \theta_{x, x x}+B_{66} \theta_{y, x x}=0, \\
& B_{11} u, x x+B_{16} v,_{x x}+D_{11} \theta_{x, x x}+D_{16} \theta_{y, x x}-A_{44}\left(w,_{x}+\theta_{x}\right)=0, \\
& B_{16} u, x x+B_{66} v,_{x x}+D_{16} \theta_{x, x x}+D_{26} \theta_{y, x x}=0, \\
& A_{44}\left(w, x x+\theta_{x, x}\right)-q=0 .
\end{aligned}
$$

The response of laminated strip can be determined completely by solving (10), (12) and (14). The terms involving derivatives of displacement $v$ and rotation $\theta_{y}$ can be eliminated from these equations using (11) and (13). An unsymmetrically laminated strip is basically a two- dimensional problem owing to the various types of material couplings. There are two possibilities, viz (i) one can solve the above mentioned three equations and later evaluate $v$ and $\theta_{y}$, or (ii) solve all the equations (10)-(14) simultaneously.

Making use of (10)-(14), $u, x x$ and $\theta_{x}$ can be expressed as follows,

$$
\begin{aligned}
u, x x & =-\beta_{1} \theta_{x, x x}-\beta_{3} \theta_{y, x x}, \\
\theta_{x} & =-w,{ }_{x}+\alpha_{1} \theta_{x, x x}+\alpha_{3} \theta_{y, x x},
\end{aligned}
$$

where,

$$
\begin{aligned}
& \beta_{1}=\frac{B_{11} A_{66}-A_{16} B_{16}}{A_{11} A_{66}-A_{16}^{2}} ; \quad \beta_{3}=\frac{B_{16} A_{66}-A_{16} B_{66}}{A_{11} A_{66}-A_{16}^{2}} \\
& \alpha_{1}=\frac{\left(D_{11} A_{66}-B_{16}^{2}\right)\left(A_{11} A_{66}-A_{16}^{2}\right)-\left(B_{11} A_{66}-B_{16} A_{16}\right)^{2}}{A_{44} A_{66}\left(A_{11} A_{66}-A_{16}^{2}\right)} \\
& \alpha_{3}=\frac{\left(D_{16} A_{66}-B_{16} B_{66}\right)\left(A_{11} A_{66}-A_{16}^{2}\right)-\left(B_{11} A_{66}-B_{16} A_{16}\right)\left(B_{16} A_{66}-B_{66} A_{16}\right)}{A_{44} A_{66}\left(A_{11} A_{66}-A_{16}^{2}\right)} .
\end{aligned}
$$

Assuming that transverse shear strains are predominantly constant, we substitute $\theta_{x}=-w, x$ and $\theta_{y}=-w, y$ on the right hand side of (18) and (19) to obtain:

$$
\begin{aligned}
u, x x x & =\beta_{1} w,_{x x x}+\beta_{3} w,_{x x y}, \\
\text { or } u & =\beta_{1} w,_{x}+\beta_{3} w,_{y}+c_{17}+c_{18} x+c_{19} y+c_{20} x y \\
\theta_{x} & =-w,_{x}-\alpha_{1} w,_{x x x}-\alpha_{3} w,_{x x y} .
\end{aligned}
$$


In (18) $C_{17}-C_{20}$ are the constants of integration and in the finite element sense they represent generalised displacement. In the absence of $\beta$ 's these coefficients allow bilinear distribution of the field $u$.

\subsection{Field for in-plane displacement $v$ and rotation $\theta_{y}$}

To derive the field for $v$ and $\theta_{y}$, equilibrium of a composite strip along the $y$ direction is considered. The equilibrium equations for this strip are simplified by dropping the terms involving derivatives with respect to $x$ from governing equations of a composite plate. Now, the procedure followed in the derivation of field for $u$ and $\theta_{x}$ is adopted to arrive at the following field description for $v$ and $\theta_{y}$.

$$
\begin{aligned}
v & =\beta_{2} w,_{y}+\beta_{4} w,_{x}+c_{21}+c_{22} x+c_{23} y+c_{24} x y, \\
\theta_{y} & =-w,,_{y}-\alpha_{2} w,_{y y y}-\alpha_{4} w, x y y
\end{aligned}
$$

where,

$$
\begin{aligned}
& \beta_{2}=\frac{B_{22} A_{66}-A_{26} B_{26}}{A_{22} A_{66}-A_{26}^{2}}, \quad \beta_{4}=\frac{B_{26} A_{66}-A_{26} B_{66}}{A_{22} A_{66}-A_{26}^{2}} \\
& \alpha_{2}=\frac{\left(D_{22} A_{66}-B_{26}^{2}\right)\left(A_{22} A_{66}-A_{26}^{2}\right)-\left(B_{22} A_{66}-B_{26} A_{26}\right)^{2}}{A_{55} A_{66}\left(A_{22} A_{66}-A_{26}^{2}\right)}, \\
& \alpha_{4}=\frac{\left(D_{26} A_{66}-B_{26} B_{66}\right)\left(A_{22} A_{66}-A_{26}^{2}\right)-\left(B_{22} A_{66}-B_{26} A_{26}\right)\left(B_{26} A_{66}-B_{66} A_{26}\right)}{A_{55} A_{66}\left(A_{22} A_{66}-A_{26}^{2}\right)} .
\end{aligned}
$$

The coefficients $C_{21}-C_{24}$ in (20) are the constants of integration and in the finite element sense they represent generalised displacement. In the absence of $\beta$ 's these coefficients allow bilinear distribution of the field $v$.

It is interesting to note that a much desired higher order polynomial approximation for in-plane displacement field $(u, v)$ in the presence of bending-extension coupling is allowed by the field derived herein. However, in the case of symmetrically laminated plates, the coefficients $\beta$ 's vanishes and the field for $u$ and $v$ reduces to bi-linear polynomial approximation. The coefficients $\alpha$ 's tend to vanish with the increase in side-to-thickness ratios and consistently satisfy the true Kirchhoff's constraints $\theta_{x}=-w, x$ and $\theta_{y}=-w, y$ in the thin regime. Therefore, the element is expected to be free of shear locking.

\subsection{Derivation of finite element equations}

To solve for the twenty-four unknowns, an additional degree of freedom $\theta_{x y}\left(=\theta_{x, y}+\theta_{y, x}\right)$ at each node is introduced apart from $u, v, w, \theta_{x}$ and $\theta_{y}$. Substitution of displacement field equations (9) and (18)-(21) into the potential function (8) and minimizing, leads to the following finite element equations:

$$
\left\{\left[\begin{array}{cc}
k_{u u} & k_{u w} \\
k_{w u} & k_{w w}
\end{array}\right]+\left[\begin{array}{cc}
0 & n 1_{u w} \\
n 1_{w u} & n 1_{w w}
\end{array}\right]+\left[\begin{array}{cc}
0 & 0 \\
0 & n 2
\end{array}\right]\right\}\left\{\begin{array}{l}
\delta_{u} \\
\delta_{w}
\end{array}\right\}+\left[\begin{array}{cc}
0 & 0 \\
0 & m
\end{array}\right]\left\{\begin{array}{l}
\ddot{\delta}_{u} \\
\ddot{\delta}_{w}
\end{array}\right\}=\left\{\begin{array}{l}
0 \\
0
\end{array}\right\}
$$


where,

$[k] \quad$ is the linear element stiffness matrix of size $24 \times 24$

$[n 1]$ is the nonlinear unsymmetric stiffness matrix of size $24 \times 24$ depending linearly on $\delta$.

$[n 2]$ is the nonlinear stiffness matrix of size $24 \times 24$ depending quadratically on $\delta$,

$[m]$ is the consistent element mass matrix of size $24 \times 24$,

$[\delta]$ is the eigenvector of size $24 \times 1$.

These elemental equilibrium equations (22) are assembled, using the standard procedures, to obtain

$$
\left\{\left[\begin{array}{cc}
K_{u u} & K_{u w} \\
K_{w u} & K_{w w}
\end{array}\right]+\left[\begin{array}{cc}
0 & N 1_{u w} \\
N 1_{w u} & N 1_{w w}
\end{array}\right]+\left[\begin{array}{cc}
0 & 0 \\
0 & N 2
\end{array}\right]\right\}\left\{\begin{array}{c}
\Delta_{u} \\
\Delta_{w}
\end{array}\right\}+\left[\begin{array}{cc}
0 & 0 \\
0 & M
\end{array}\right]\left\{\begin{array}{l}
\ddot{\Delta}_{u} \\
\ddot{\Delta}_{w}
\end{array}\right\}=\left\{\begin{array}{l}
0 \\
0
\end{array}\right\} .
$$

To compute the nonlinear frequencies, the linear eigenvalue problem is solved as a first step. The eigenvector corresponding to the fundamental frequency is assumed as the spatial distribution and nonlinear finite element equations are reduced to a single nonlinear secondorder ordinary differential equation following the procedure given by Singh et al (1991). The nonlinear equation so obtained is of the following form,

$$
\ddot{A}+\alpha A+\beta A^{2}+\gamma A^{3}=0,
$$

where $A$ denotes maximum spatial deflection at any instant of time and $\alpha, \beta$ and $\gamma$ are coefficients of linear and nonlinear stiffnesses and are computed as follows,

$$
\begin{aligned}
& \alpha=\{\Delta\}^{T}\left\{\left[K_{w w}\right]-\left[K_{w u}\right]\left[K_{u u}\right]^{-1}\left[K_{u w}\right]\right\}\{\Delta\}, \\
& \beta=\{\Delta\}^{T}\left\{\left[N 1_{w w}\right]-\left[K_{w u}\right]\left[K_{u u}\right]^{-1}\left[N 1_{u w}\right]-\left[N 1_{w u}\right]\left[K_{u u}\right]^{-1}\left[K_{u w}\right]\right\}\{\Delta\}, \\
& \gamma=\{\Delta\}^{T}\left\{\left[N 2_{w w}\right]-\left[N 1_{w u}\right]\left[K_{u u}\right]^{-1}\left[N 1_{u w}\right]\right\}\{\Delta\} .
\end{aligned}
$$

The nonlinear sub-matrices required above are computed using linear normalised eigenvector.

Equation (24) is solved by employing the Direct Numerical Integration Method (Singh et al 1991) to compute the nonlinear frequencies/periods as follows:

$$
\begin{aligned}
\frac{T}{2}= & \frac{\pi}{\omega}=\int_{0}^{\pi / 2} \mathrm{~d} \theta /\left\{\alpha\left[1+\frac{2}{3} \frac{\beta}{\alpha} F_{1}(\theta) A_{\max }+\frac{1}{2} \frac{\gamma}{\alpha} F_{2}(\theta) A_{\max }^{2}\right]\right\}^{1 / 2} \\
& +\int_{0}^{\pi / 2} \mathrm{~d} \theta /\left\{\alpha\left[1-\frac{2}{3} \frac{\beta}{\alpha} F_{1}(\theta) B_{\max }+\frac{1}{2} \frac{\gamma}{\alpha} F_{2}(\theta) B_{\max }^{2}\right]\right\}^{1 / 2}
\end{aligned}
$$

where, $F_{1}(\theta)=\left(1+\sin \theta+\sin ^{2} \theta\right) /(1+\sin \theta)$ and $F_{2}(\theta)=1+\sin ^{2} \theta . A_{\max }$ and $B_{\max }$ represent amplitudes of positive and negative deflection half-cycles. In the present analysis for a particular $A_{\max }, B_{\max }$ is computed using the principle of energy conservation (for more details see Singh et al 1991). The integrands in (24) are computed numerically by employing the 5-point Gauss quadrature formula. For the nonlinear period/frequency, (24) is derived using the initial conditions: $A=A_{\max }$ and $\dot{A}=0$ at $t=0$. 
Table 1. Material properties used in the present study.

\begin{tabular}{lccccc}
\hline Material & $E_{L} / E_{T}$ & $G_{L T} / E_{T}$ & $G_{L Z} / E_{T}$ & $G_{T Z} / E_{T}$ & $v_{L T}$ \\
\hline M-I & & Isotropic & & & 0.3 \\
M-II & 25.0 & 0.5 & 0.5 & 0.2 & 0.25 \\
\hline
\end{tabular}

\section{Numerical results and discussions}

To validate the material finite element (MFE) developed in the preceding section, a series of numerical examples involving effects of material coupling, transverse shear flexibility and boundary conditions are solved. Linear frequencies of various plate configurations computed using MFE are compared with those obtained using quadrilateral four-noded elements (QUAD4) based on the selective integration scheme (Bathe 1990). Particular emphasis is placed on establishing the effects of anisotropy, shear deformation and edge restraints on the rate of convergence and accuracy with mesh refinement of the MFE element. Throughout this section, numerical results are obtained by idealizing the whole plate. The nonlinear frequencies for various plate configurations are computed using the direct numerical integration method. The fundamental mode shape corresponding to $8 \times 8$ mesh discretization over the whole plate is used to compute $\alpha, \beta$ and $\gamma$, (24).

Mechanical properties in the form of moduli ratios and geometric edge constraints considered in this study are given in tables 1 and 2 respectively.

\subsection{Validation of material finite element}

4.1a Homogeneous isotropic square plates: Homogeneous isotropic square plates with all edges simply-supported (SSSS) and clamped (CCCC) are discretized with progressively refined meshes, i.e. $2 \times 2,4 \times 4,6 \times 6, \ldots 32 \times 32$ of MFE and QUAD4 elements. The variation of non-dimensional frequency parameter $\lambda_{\omega 0}$ corresponding to the fundamental frequency $\omega_{0}$ with mesh refinement for various side to thickness ratios $(a / t=5,10,100)$ is presented in tables 3 and 4 . The results indicate that the rate of convergence of the frequency parameter of the proposed MFE is far superior compared to the QUAD4 element, especially so for thin plates. In fact, in the case of thin plates with all edges clamped, the authors found that even a $32 \times 32$ mesh of QUAD4 elements over the whole plate does not yield converged frequencies. The slow convergence of QUAD4 elements in the case of thin plates is an established problem (see Lakshminarayana \& Sridhara Murthy 1984), whose solution in the formal manner has been sought for long. The proposed element, being nonconforming, is found to converge from the top in the case of SSSS plates and from the bottom for CCCC plates.

$4.1 \mathrm{~b}$ Four-layer symmetric cross-ply $\left[0^{\circ} / 90^{\circ}\right]_{s}$ plates: Comparison of the frequency parameter convergence characteristics of MFE with QUAD4 elements for square simplysupported (SSSS) four-layered symmetric cross-ply plates is presented in table 5. As in the preceding study, the proposed MFE converges much faster, especially when the plates are thin, than the conventional QUAD4 elements. The study also indicates that the convergence of MFE in case of thin plates is faster when compared to thick plates, while the reverse is true for QUAD4 elements. 
Table 2. Boundary conditions used in the present study.

\begin{tabular}{lcc}
\hline Boundary condition & \multicolumn{1}{c}{ Edge $x=0, a$} & Edge $y=0, b$ \\
\hline Simple support (SSSS) & $u=v=w=\theta_{y}=0$ & $u=v=w=\theta_{x}=0$ \\
Clamped (CCCC) & $u=v=w=\theta_{x}=\theta_{y}=0$ & $u=v=w=\theta_{x}=\theta_{y}=0$ \\
\hline
\end{tabular}

Table 3. Comparison of frequency parameter and convergence characteristics of MFE with QUAD4 elements for square simply-supported isotropic plates.

\begin{tabular}{|c|c|c|c|c|c|c|}
\hline \multirow[b]{3}{*}{ Mesh size } & \multicolumn{6}{|c|}{$\lambda_{\omega 0}=\left(\rho \omega_{0}^{2} b^{4}\right) /\left(E t^{2}\right)$} \\
\hline & \multicolumn{2}{|c|}{$a / t=100$} & \multicolumn{2}{|c|}{$a / t=10$} & \multicolumn{2}{|c|}{$a / t=5$} \\
\hline & MFE & QUAD4 & MFE & QUAD4 & MFE & QUAD4 \\
\hline $2 \times 2$ & 35.8243 & 64.5259 & 34.1275 & 56.8780 & 29.6728 & 42.418 \\
\hline $4 \times 4$ & 35.6706 & 41.0112 & 33.6378 & 37.9195 & 28.3612 & 31.107 \\
\hline $8 \times 8$ & 35.6608 & 36.9068 & 33.4251 & 34.3782 & 28.0293 & 28.657 \\
\hline $12 \times 12$ & 35.6600 & 36.2046 & 33.3606 & 33.7659 & 27.9531 & 28.223 \\
\hline $16 \times 16$ & 35.6600 & 35.9629 & 33.3600 & 33.5546 & 27.9500 & 28.073 \\
\hline $32 \times 32$ & 35.6600 & 35.7317 & 33.3600 & 33.3522 & 27.9500 & 27.928 \\
\hline
\end{tabular}

Table 4. Comparison of frequency parameter and convergence characteristics of MFE with QUAD4 elements for square clamped-clamped isotropic plates.

\begin{tabular}{|c|c|c|c|c|c|c|}
\hline \multirow[b]{3}{*}{ Mesh size } & \multicolumn{6}{|c|}{$\lambda_{\omega 0}=\left(\rho \omega_{0}^{2} b^{4}\right) /\left(E t^{2}\right)$} \\
\hline & \multicolumn{2}{|c|}{$a / t=100$} & \multicolumn{2}{|c|}{$a / t=10$} & \multicolumn{2}{|c|}{$a / t=5$} \\
\hline & MFE & QUAD4 & MFE & QUAD4 & MFE & QUAD4 \\
\hline $2 \times 2$ & 106.1268 & 57692.32 & 87.0956 & 576.9232 & 56.4345 & 144.2307 \\
\hline $4 \times 4$ & 112.9132 & 169.4962 & 89.4520 & 128.9878 & 57.8923 & 78.3457 \\
\hline $8 \times 8$ & 117.5508 & 128.4983 & 95.2449 & 103.6643 & 63.5807 & 67.5621 \\
\hline $12 \times 12$ & 118.0777 & 122.6680 & 96.4621 & 99.8032 & 64.2466 & 65.7950 \\
\hline $16 \times 16$ & 118.1000 & 120.7274 & 96.6521 & 98.5046 & 64.3200 & 65.1911 \\
\hline $32 \times 32$ & 118.1000 & 118.9049 & 96.7000 & 97.2743 & 64.3200 & 64.6160 \\
\hline
\end{tabular}

Table 5. Comparison of frequency parameter and convergence characteristics of MFE with QUAD4 elements for four-layer symmetric cross-ply $\left[0^{\circ} / 90^{\circ} / 90^{\circ} / 0^{\circ}\right]$ square plates with all edges clamped.

\begin{tabular}{|c|c|c|c|c|c|c|}
\hline \multirow[b]{3}{*}{ Mesh size } & \multicolumn{6}{|c|}{$\lambda_{\omega 0}=\left(\rho \omega_{0}^{2} b^{4}\right) /\left(E_{T} t^{2}\right)$} \\
\hline & \multicolumn{2}{|c|}{$a / t=100$} & \multicolumn{2}{|c|}{$a / t=10$} & \multicolumn{2}{|c|}{$a / t=5$} \\
\hline & MFE & QUAD4 & MFE & QUAD4 & MFE & QUAD4. \\
\hline $2 \times 2$ & 232.6449 & 445.5498 & 162.8036 & 212.2106 & 87.8789 & 89.9776 \\
\hline $4 \times 4$ & 230.7619 & 268.9059 & 150.4781 & 164.9298 & 78.4956 & 81.2346 \\
\hline $8 \times 8$ & 230.5751 & 239.4447 & 149.9645 & 153.2755 & 77.4294 & 77.9764 \\
\hline $12 \times 12$ & 230.5554 & 234.4497 & 149.8054 & 151.1732 & 77.1402 & 77.3520 \\
\hline $16 \times 16$ & 230.5539 & 232.7333 & 149.8054 & 150.8095 & 77.1323 & 77.1323 \\
\hline $32 \times 32$ & 230.5539 & 231.0932 & 149.8054 & 149.7405 & 77.1323 & 76.9199 \\
\hline
\end{tabular}


Table 6. Comparison of frequency parameter and convergence characteristics of MFE with QUAD4 elements for two layer anti-symmetric angle-ply $\left[45^{\circ}-45^{\circ}\right]$ square plates with all edges simply-supported.

\begin{tabular}{|c|c|c|c|c|c|c|}
\hline \multirow[b]{3}{*}{ Mesh size } & \multicolumn{6}{|c|}{$\lambda_{\omega 0}=\left(\rho \omega_{0}^{2} b^{4}\right) /\left(E_{T} t^{2}\right)$} \\
\hline & \multicolumn{2}{|c|}{$a / t=100$} & \multicolumn{2}{|c|}{$a / t=10$} & \multicolumn{2}{|c|}{$a / t=5$} \\
\hline & MFE & QUAD4 & MFE & QUAD4 & MFE & QUAD4 \\
\hline $2 \times 2$ & 250.2426 & 723.5212 & 190.2979 & 304.6229 & 106.3183 & 111.0599 \\
\hline $4 \times 4$ & 238.8757 & 294.5394 & 173.0777 & 192.2091 & 93.6526 & 95.2542 \\
\hline $8 \times 8$ & 236.0450 & 247.9208 & 167.5264 & 170.9820 & 89.2614 & 89.6709 \\
\hline $12 \times 12$ & 235.4093 & 240.4081 & 165.9305 & 167.3103 & 88.4573 & 88.6145 \\
\hline $16 \times 16$ & 235.4000 & 237.8473 & 165.3000 & 166.0413 & 88.3000 & 88.2421 \\
\hline $32 \times 32$ & 235.4000 & 235.4107 & 165.3000 & 164.8258 & 88.3000 & 87.8808 \\
\hline
\end{tabular}

4.1c Two-layer antisymmetric cross- and angle-ply square plates: The plate configurations considered so far did not involve material coupling $\left(\beta_{i}=0\right)$, therefore the in-plane and out-of-plane responses were uncoupled. The response was governed by the transverse displacement and two bending rotations. Therefore, the only non-zero coupling coefficients in the displacement field were $\alpha$ 's (refer (19) and (21)). However, in the case of antisymmetric cross- and angle-ply plates, the in-plane and out-of-plane responses are coupled. Therefore the $\beta$ 's in the displacement field equations (18) and (20) are non-zero. These non-zero $\beta$ 's allow higher-order in-plane displacement field descriptions and hence help in accelerating the convergence. The comparison of convergence characteristics of frequency parameter of such plates obtained using MFE and QUAD4 is presented in tables 6 and 7. The study once again indicates that MFE performs much better than QUAD4 even for unsymmetrically laminated plates.

\subsection{Large amplitude vibration analysis}

The eigenvector corresponding to the fundamental frequency obtained using $8 \times 8$ mesh over the whole plate is assumed as spatial distribution herein and in the rest of the section to obtain the linear and nonlinear stiffness coefficients of (24). Nonlinear to linear frequency

Table 7. Comparison of frequency parameter and convergence characteristics of MFE with QUAD4 elements for two layer anti-symmetric cross-ply $\left[0^{\circ} / 90^{\circ}\right]$ square plates with all edges simply-supported.

\begin{tabular}{|c|c|c|c|c|c|c|}
\hline \multirow[b]{3}{*}{ Mesh size } & \multicolumn{6}{|c|}{$\lambda_{\omega 0}==\left(\rho \omega_{0}^{2} b^{4}\right) /\left(E_{T} t^{2}\right)$} \\
\hline & \multicolumn{2}{|c|}{$a / t=100$} & \multicolumn{2}{|c|}{$a / t=10$} & \multicolumn{2}{|c|}{$a / t=5$} \\
\hline & MFE & QUAD4 & MFE & QUAD4 & MFE & QUAD4 \\
\hline $2 \times 2$ & 154.1471 & 447.0983 & 120.2477 & 240.7447 & 72.7379 & 101.1085 \\
\hline $4 \times 4$ & 170.3886 & 219.1110 & 124.5389 & 157.2102 & 73.8057 & 85.6305 \\
\hline $8 \times 8$ & 176.2747 & 187.3778 & 132.4640 & 140.1335 & 77.8899 & 80.2999 \\
\hline $12 \times 12$ & 177.3314 & 182.1677 & 134.0895 & 137.1721 & 78.3765 & 79.2986 \\
\hline $16 \times 16$ & 177.3000 & 180.3877 & 134.0900 & 136.1504 & 78.4000 & 78.9477 \\
\hline $32 \times 32$ & 177.3000 & 178.6941 & 134.0900 & 135.1743 & 78.4000 & 78.6091 \\
\hline
\end{tabular}




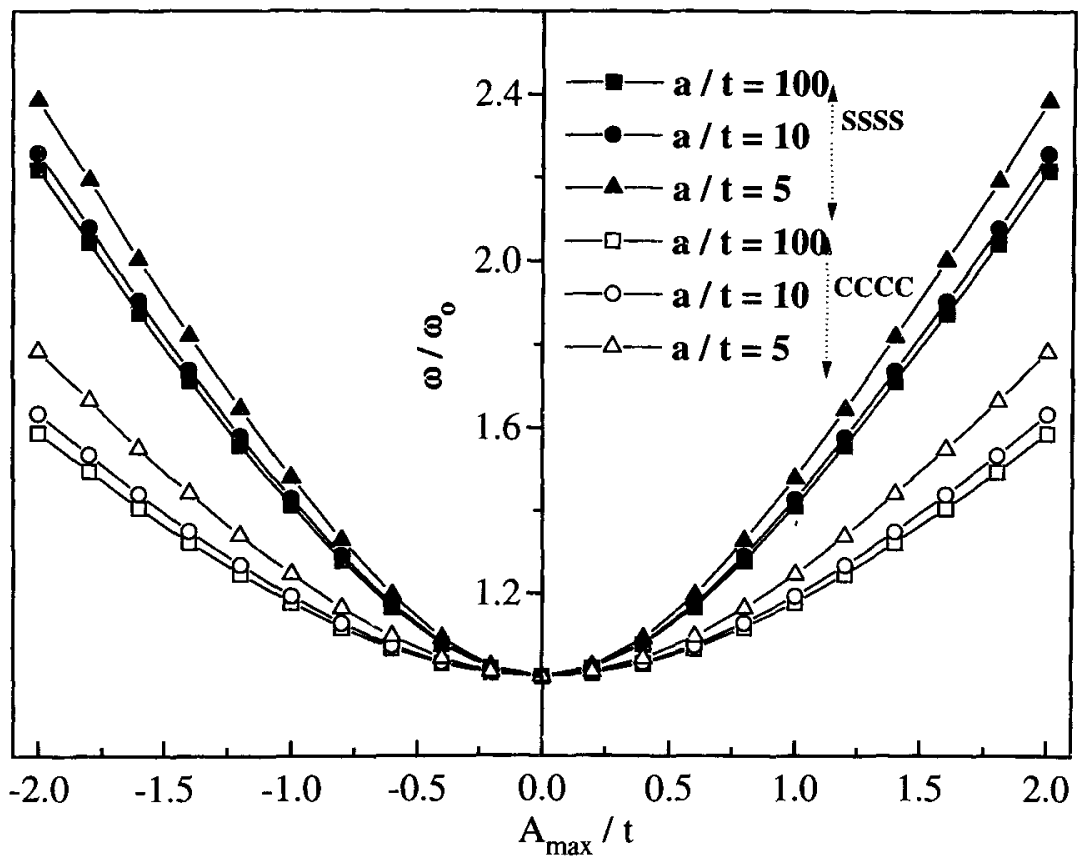

Figure 2. Variation of frequency ratio with amplitude for square isotropic plates.

ratios $\left(\omega / \omega_{0}\right)$ at different amplitudes are computed using the Direct Numerical Integration Method for various plate configurations.

4.2a Homogeneous isotropic square plates: The variation of nonlinear to linear frequency ratios $\left(\omega / \omega_{0}\right)$ with amplitude ratio $\left(A_{\max } / t\right)$ for various side to thickness ratios is plotted in figure 2 . The edge conditions considered in this study are (1) all edges simplysupported, and (2) all edges clamped. The results indicate that frequency increases with increase in amplitude and decrease in side-to-thickness ratio. Plates with SSSS edge conditions exhibit more nonlinearity than plates with $\mathrm{CCCC}$ edge conditions.

$4.2 b \quad$ Two-layer cross- and angle-ply plates: The nonlinear stiffness coefficient $\beta$ in (24) is zero for the preceding as well as the present study. For isotropic plates, it vanishes since bending-extension coupling coefficients $B_{i j}$ are zero. However, in the particular case of square antisymmetric cross-ply and even rectangular antisymmetric angle-ply plates $\beta$ is zero. Thus, the governing equation (24) is typically a Duffing's equation, whose solution can be found by either the perturbation method or the direct numerical integration method. The mechanical properties of material-II are considered in the studies shown in figures 3 and 4 . The study presented in figure 3 indicates that two-layer cross-ply plates with simplysupported edge conditions exhibit higher nonlinearity compared to two-layer angle-ply plates.

Similar results for rectangular $(a / b=1.2)$ two-layer antisymmetric cross- and angleply plates with simply-supported edges are plotted in figure 4 . It is seen that for rectangular antisymmetric cross-ply plates, $\beta$ in (18) does not vanish. Therefore such plates oscillate with different amplitudes in positive- and negative-deflection half-cycles, while 


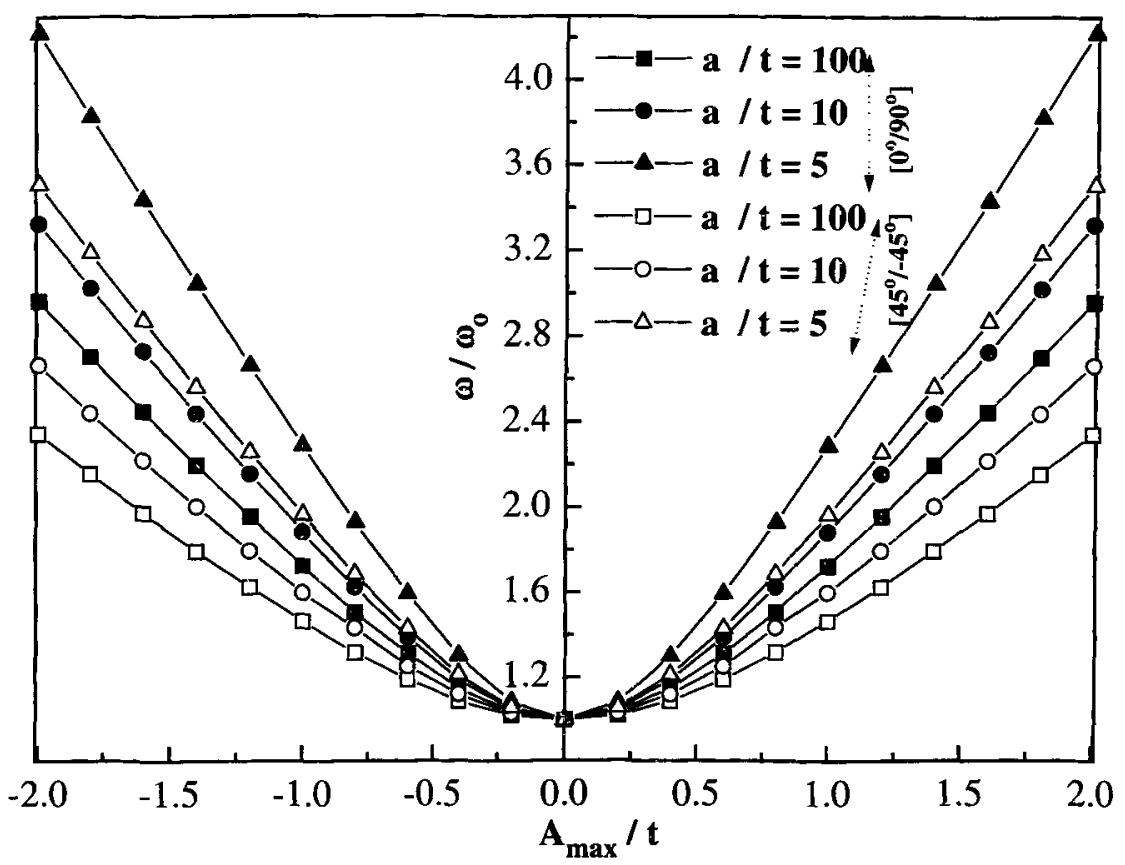

Figure 3. Variation of frequency ratio with amplitude for square simply supported two layer cross- and angle-ply plates.

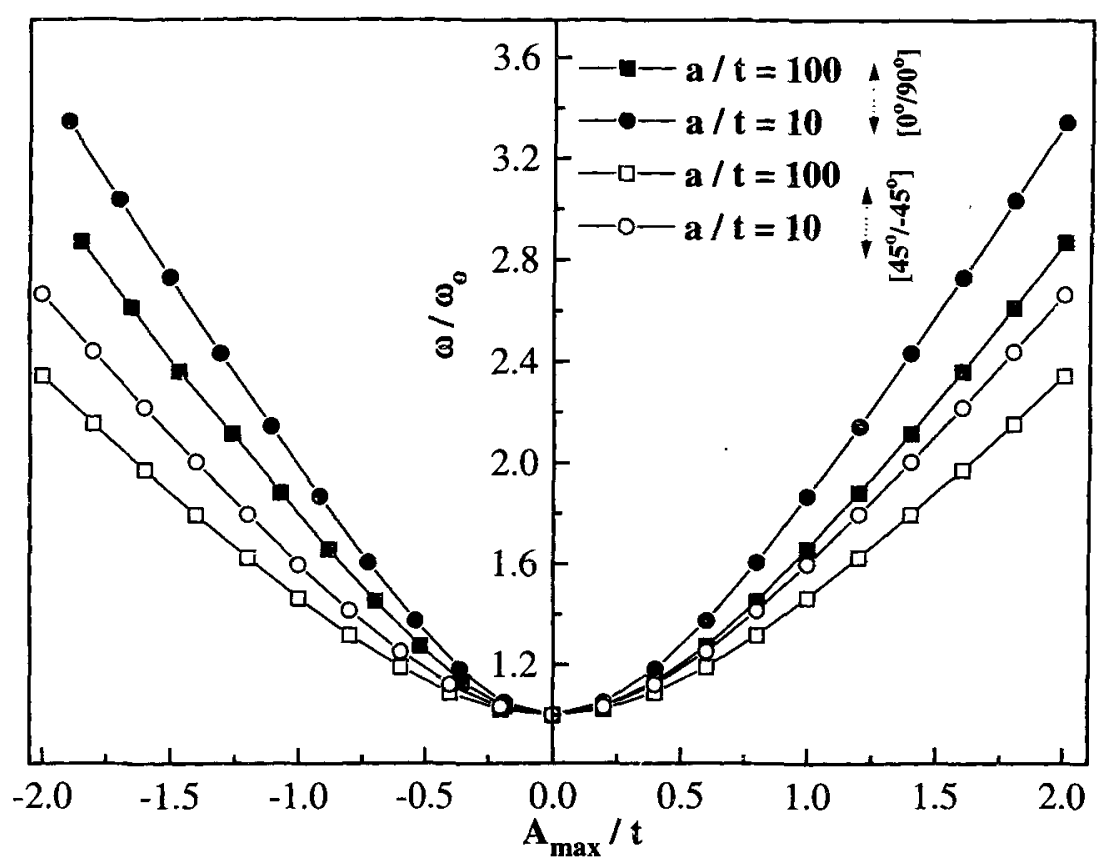

Figure 4. Variation of frequency ratio with amplitude for rectangular $(a / b=1.2)$ simply supported cross- and angle-ply plates. 
antisymmetric angle-ply plates oscillate with the same amplitude in positive- and negativedeflection half-cycles.

\section{Conclusions}

An accurate simple four-node shear flexible composite plate element based on a coupled displacement field is proposed in this paper for investigating the large amplitude vibration behaviour of composite plates. The displacement field for the proposed MFE is derived from equilibrium considerations, and hence not only depends on the element coordinates but on the material properties as well. The rate of convergence of QUAD4 elements is very slow when the plate lay-ups are asymmetric or the plate is thin, whereas the proposed MFE continues to converge faster irrespective of the plate lay-up and the side-to-thickness ratio. The element employs full Gaussian integration rules for computing stiffness and mass matrices, and is free from shear locking and any spurious modes. The displacement field of the MFE changes with lay-up secience resulting in a non-conforming but highly efficient and accurate element. The direct numerical integration method employed herein does not assume temporal variation and yields highly accurate solutions. It is found that rectargular $(a / b \neq 1)$ antisymmetric cross-ply plates oscillate with different amplitudes in positiveand negative-deflection half-cycles.

\section{References}

Ahmed S, Irons B M, Zienkiewicz O C 1970 Analysis of thick and thin structures by curved finite elements. Comput. Methods Appl. Mech. Eng. 50: 121-145

Bathe K J 1990 Finite element procedures in engineering analysis (Englewood Cliffs, NJ: PrenticeHall)

Bogner F K, Fox R L, Schmit L A 1966 The generation of inter-element compatible stiffness and mass matrices by the use of interpolation formulas. Presented at the Wright-Patterson Air Force Base, Ohio Meeting on Matrix Methods in Structural Mechanics, AFFDL-TR-66-80, pp 397-443

Crisfield M A 1984 A quadratic Mindlin element using shear constraints. Comput. Struct. 18: $833-852$

Hinton E, Campbell J S 1974 Local and global smoothing of discontinuous finite element functions using a least squares method. Int. J. Numer. Methods Eng. 8: 461-480

Huang S 1998 Nonlinear vibrations of a hinged orthotropic circular plate with a concentric rigid mass. J. Sound Vibr. 214: 873-884

Hughes T J R, Tezduyar T E 1981 Finite elements based upon Mindlin plate theory, with particular reference to the four-node bilinear isoperimetric element. J. Appl. Mech. 48: 587-596

Hughes T J R, Taylor R L, Kanoknukulchai W 1977 A simple and efficient finite element for plate bending. Int. J. Numer. Methods Eng. 11: 1529-1543

Lakshminarayana H V, Sridhara Murthy S 1984 A shear-flexible triangular finite element model for laminated composite plates. Int. J. Numer. Methods Eng. 30: 591-623

Lee S W, Pian T H H 1978 Improvement of piate and shell finite elements by mixed formulations. AIAA J. 16: 29-34

Leung A Y T, Chui S K 1997 Nonlinear vibration of the von Karman square plate by IHB method. J. Sound Vibr. 204: 239-247

Nayfeh A H, Mook D T 1979 Nonlinear oscillations (New York: John Wiley)

Noor A K, Anderson C M 1977 Mixed isoparimetric finite element models of laminated composite shells. Comput. Methods Appl. Mech. Eng. 11: 255-280

Pawsey S F, Clough R W 1971 Improved numerical integration of thick shell finite elements. Int. J. Numer. Methods Eng. 3: 575-586 
Prathap G 1984 An optimally constrained 4-noded quadrilateral thin plate bending element. Comput. Struct. 18: 789-794

Prathap G 1985 A $C^{0}$ continuous 4-noded cylindrical shell element. Comput. Struct. 21: 995-999

Pugh E D L, Hinton E, Zienkiewicz O C 1978 A study of quadrilateral plate bending elements with reduced integration. Int. J. Numer. Methods Eng. 12: 1059-1079

Qaisi M I 1997 A power series solution for the nonlinear vibrations of beams. J. Sound Vibr. 199: $587-594$

Sathymoorthy M 1982a Nonlinear analysis of beams, Part II: Finite element method. Shock Vibr. Dig. 14: 7-18

Sathymoorthy M 1982b Nonlinear analysis of beams, Part I: A survey of recent advances. Shock Vibr. Dig. 14: 19-35

Sathymoorthy M 1987 Nonlinear vibration analysis of plates: A review and survey of current development. Appl. Mech. Rev. 40: 1553-1561

Singh G, Venkateswara Rao G 1998 Nonlinear oscillations of thick asymmetric cross-ply beams. Acta Mech. 127: 135-146

Singh G, Venkateswara Rao G, Iyengar N G R 1991 Analysis of nonlinear vibrations of unsymmetrically laminated composite beams. AIAA J. 29: 1727-1735

Singh G, Venkateswara Rao G, Iyengar N G R 1993 Large deflection behaviour of shear deformable composite plates using a simple higher order theory. Compos. Eng. 3: 507-525

Somashekar B R, Prathap G, Ramesh Babu C 1987 A field consistent four-noded laminated anisotropic plate/shell element. Comput. Struct. 25: 345-353

Spilker R L, Munir N I 1980 Improvement of plate and shell finite elements by mixed formulations. Int. J. Numer: Methods Eng. 15: 1239-1260

Stoker J J 1950 Nonlinear vibrations in mechanical and electrical systems (New York: Interscience)

Yang P C, Norris C M, Stavsky 1966 Elastic wave propagation in heterogeneous plates. Int. J. Solid Struct. 2: 665-684

Zienkiewicz O C 1977 The finite element method 3rd edn (London: McGraw-Hill)

Zienkiewicz O C, Taylor R L, Too J M 1971 Reduced integration techniques in general analysis of plates and shells. Int. J. Numer. Methods Eng. 3: 275-290 